\title{
Avaliação Empírica do Modelo de Burgstahler e Dichev no Mercado Brasileiro
}

\author{
Daphnis Theodoro da Silva Jr. \\ Alexsandro Broedel Lopes \\ Luiz João Corrar
}

\section{RESUMO}

Estudos recentes indicam que lucros e valor patrimonial são importantes fatores explicativos do valor de mercado das ações de empresas. Esta realização se fundamenta no trabalho de Burgstahler e Dichev (1997) que, a partir de um modelo de avaliação estruturado com base nos modelos de precificação de opções, concluiu que o valor de uma ação é uma função convexa do lucro, quando o valor patrimonial é mantido constante e, simultaneamente, o valor de uma ação é função convexa do valor patrimonial, quando o lucro é mantido constante. Empregando metodologia adaptada daquela utilizada no trabalho de Burgstahler e Dichev (1997) e utilizando uma amostra composta por ações representativas do mercado brasileiro, os testes realizados por meio da comparação de duas carteiras de ações, uma com alta e outra com baixa razão preço/valor patrimonial, geraram resultados que corroboram a relação supramencionada.

Palavras-chave: avaliação de empresas; precificação de opções; valor residual.

\begin{abstract}
Recent studies indicate that earnings and book values are important factors in explaining stock prices. This paper uses the work of Burgstahler and Dichev (1997), which used a model based on option pricing theory and concluded that stock prices are convex functions of profits when book values are kept constant and stock prices are a convex function of book values when earnings are constant. Using an adapted methodology and applying on a sample of stocks traded on the Brazilian market, the tests realized through a comparison of two portfolios with high and low price-to-book ratio supported the hypothesis, as expected.
\end{abstract}

Key words: valuation; option pricing; residual income. 


\section{INTRODUÇÃO}

A busca da compreensão de quais variáveis são relevantes, bem como da forma pela qual interagem na formação do preço de mercado das ações, é tema há muito estudado. Na pesquisa contábil, modelos de avaliação de ações têm sido usados para a verificação da relevância dos dados contábeis na explicação do preço das ações. Basicamente as variáveis contábeis utilizadas são o lucro e o valor do patrimônio líquido das empresas.

Sob o enfoque do lucro, em artigo de 1959, Modigliani e Miller discutiram as vantagens da utilização dos dividendos em lugar dos lucros, afirmando que estes estariam sujeitos à variações de curto prazo, enquanto aqueles representariam a real capacidade de geração de retornos no longo prazo. Em trabalho posterior, Modigliani e Miller (1961, p. 415) identificaram na literatura pelo menos quatro abordagens razoavelmente diferentes de avaliação de empresas, que são: (i) a abordagem do fluxo de caixa descontado ( $D C F$ - discounted cash flow); (ii) a abordagem dos rendimentos correntes, mais as oportunidades futuras de investimento ou crescimento $(C E+G O-$ current earnings + growth oportunities); (iii) a abordagem do fluxo de dividendos (DDM - dividend discount model); (iv) a abordagem do fluxo de rendimentos (stream of earnings); ainda estes autores demonstraram que, numa economia ideal, caracterizada por mercados de capitais perfeitos, comportamento racional e perfeita certeza, os valores resultantes serão equivalentes para as quatro abordagens, porém fazem ressalva quanto à última abordagem, afirmando (Modigliani \& Miller, 1961, p. 420):

Infelizmente, é também extremamente fácil errar ou interpretar erroneamente o enfoque do fluxo de rendimentos, como seria o caso se o valor da empresa fosse definido como simplesmente a soma descontada dos rendimentos totais futuros. ... Em verdade, deveríamos afirmar que não há nenhum trabalho, de nosso conhecimento, que tenha seriamente avançado nesta abordagem.

De fato, tanto modelos de desconto do fluxo esperado de dividendos quanto de desconto do fluxo esperado de lucros são utilizados até os dias atuais.

Segundo Watts e Zimmerman (1986, p. 37), a popularização da pesquisa positiva em contabilidade teve início com o trabalho de Ball e Brown (1968) que, da mesma forma que outros, publicados posteriormente (Beaver, Clark, \& Wright, 1979; Collins \& Kothari, 1989; Barth, Beaver, \& Landsman, 1992; entre outros) 
mostram que o preço de mercado das ações tem relação com os lucros correntes das empresas emissoras dessas ações. O trabalho de Lipe (1990) mostra que essa relação varia em função da persistência dos resultados ao longo do tempo.

Nichols e Wahlen (2004) apresentam uma revisão da literatura relativa à pesquisa clássica em contabilidade sob o enfoque de como os resultados das empresas se relacionam com o retorno de suas ações e, utilizando dados relativos ao período compreendido entre 1998 e 2002, mostram que os resultados reportados pelos estudos de Ball e Brown (1968), Kormendi e Lipe (1987) e Bernard e Thomas (1989) são também válidos para os dados desse novo período. Por outro lado, Bamber, Christensen e Gaver (2000), usando como base o trabalho de Beaver (1968), mostram a influência da forma como o planejamento e o desenvolvimento de um trabalho de pesquisa pode influir nos seus resultados, e destacam os cuidados que devem ser tomados quanto à generalização dos mesmos.

Abordando a questão da pesquisa contábil no mercado de capitais do ponto de vista econométrico, Kothari e Zimmerman (1995) discutem as implicações da escolha entre a utilização dos preços das ações ou de seus retornos nos modelos empregados.

Sob o enfoque do valor do patrimônio líquido, segundo Burgstahler e Dichev (1997, p. 188), na mesma época vários trabalhos mostraram que o valor das ações é relacionado a dados do balanço patrimonial, como ativos e passivos (por exemplo: Barth, 1991; Landsman, 1986). Estes referidos estudos fazem inferências sobre o valor de uma empresa a partir dos valores de seus ativos e passivos, considerando o pressuposto de que esses valores definem os resultados futuros dessa empresa.

O efeito da combinação dos lucros com o valor do patrimônio líquido na estimação do valor das ações foi estudado empiricamente por Bernard (1995) e teoricamente por Ohlson (1995). O modelo proposto por Ohlson (1995) relaciona o preço de uma ação à combinação de seu valor patrimonial, lucro e dividendos, gerando uma função linear aditiva de valor patrimonial e lucros anormais. O trabalho de Ohlson (1995) representa uma estrutura teórica de grande interesse para pesquisas empíricas que, no Brasil, foram exploradas por Lopes (2001) e Oliveira (2001).

Penmam (1998), cuja abordagem foi discutida por Burgstahler (1998), procurou encontrar os pesos para que a ponderação entre os resultados reportados por uma empresa e o valor patrimonial das suas ações propiciasse a estimação do valor de mercado das suas ações. 
Segundo Kothari (2001), as principais fontes de demanda da pesquisa contábil relativa ao mercado de capitais são a análise fundamentalista e a avaliação, testes relativos à eficiência de mercado, e o papel dos números contábeis nos contratos e no processo político. O principal objetivo desse trabalho é oferecer hipóteses que competem na explicação dos resultados observados. O referido trabalho de Kothari (2001), bem como o livro Financial Reporting: An Accounting Revolution (Beaver, 1998) são ricas fontes de referências sobre pesquisas realizadas na área. Lee (2001), em trabalho escrito com o objetivo de discutir o Kothari (2001), acrescenta à discussão aspectos relativos ao que chamou de 'visão ingênua da eficiência de mercado'.

Libby, Bloomfield e Nelson (2002) utilizam enfoques da teoria comportamental e da economia experimental na pesquisa contábil relativa à troca de informações entre os participantes do mercado, representados, em última análise, pelos geradores de informação, os auditores e os investidores.

O objetivo deste trabalho é testar no mercado acionário brasileiro a proposição teórica formulada, e testada com dados do mercado americano, por Burgstahler e Dichev (1997), no sentido de que o valor de mercado da ação de uma empresa é função convexa do lucro e do valor patrimonial dessa empresa.

A argumentação utilizada por Burgstahler e Dichev (1997, p. 188) parte do pressuposto de que o valor patrimonial provê informação sobre o valor líquido dos recursos da empresa, e que o lucro fornece uma medida de valor que reflete os resultados específicos dessa empresa no emprego desses recursos. Dessa forma os lucros representam o determinante mais importante do valor da empresa, quando suas atividades correntes são bem sucedidas e existe a expectativa de que continuem assim no futuro; esta situação é observada, quando a relação preço / valor patrimonial é alta. Por outro lado, o valor patrimonial é mais importante, quando os recursos da empresa provavelmente serão adaptados para usos alternativos superiores, sendo esta situação observada quando a relação preço / valor patrimonial é baixa.

De maneira geral, o valor da empresa é representado por uma ponderação entre seu lucro e seu valor patrimonial, pois a mesma possui sempre a opção de continuar suas atividades correntes ou adaptar seus recursos a usos alternativos. $\mathrm{O}$ modelo proposto pelos referidos autores explicitamente reconhece que o potencial de adaptação dos recursos da empresa representa uma opção disponível para a empresa, e que o valor dessa opção deve ser refletido em seu valor de mercado. A empresa é vista como a combinação de um conjunto de recursos e uma tecnologia específica de seu negócio, onde essa tecnologia 
específica é definida como a forma com que essa empresa utiliza seus recursos para produzir um fluxo de resultados. Nesse contexto os dois elementos complementares do valor da empresa são o 'valor dos recursos em operação' (recursion value) e o 'valor de adaptação' (adaptation value). O 'valor dos recursos em operação' (recursion value) é o valor resultante do desconto do fluxo de resultados futuros esperados considerando como pressuposto que a empresa continuará a aplicar sua corrente tecnologia de negócio aos seus recursos. O 'valor de adaptação' (adaptation value) é o valor dos recursos da empresa independentemente da corrente tecnologia de negócios empregada, sendo que esse valor existe sempre que os recursos podem ser adaptados a usos alternativos.

Os usos alternativos podem envolver adaptações externas, quando os recursos são vendidos; ou internas, quando os recursos são adaptados para outros usos. O conceito de 'valor de adaptação' (adaptation value) utilizado por Burgstahler e Dichev (1997) está em linha com a discussão de Wright (1967) sobre o papel do balanço patrimonial como provedor de informações relevantes para o mercado de capitais. Nesse trabalho Wright (1967) argumenta que os dados contidos no balanço patrimonial são informativos quanto ao valor de adaptação e que esse valor de adaptação pode ser tanto o valor de venda quanto o custo de reposição, dependendo de que tipo de adaptação seja mais provável, a externa ou a interna. Pelo fato de a empresa possuir a opção de adaptar seus recursos a usos distintos dos correntes, o valor de suas ações reflete ambos os valores, o 'valor de adaptação' e o 'valor dos recursos em operação'. Burgstahler e Dichev (1997) combinam lucro e valor patrimonial em um modelo de avaliação de opções para o valor da ação, em que a probabilidade de exercício do 'valor de adaptação' depende dos valores relativos do valor patrimonial e do lucro. A principal predição desse modelo de avaliação é sua convexidade, ou seja, (i) o valor da ação é função convexa do lucro, quando o valor patrimonial é mantido constante e, simultaneamente, (ii) o valor da ação é função convexa do valor patrimonial, quando o lucro é mantido constante. Os testes empíricos realizados pelos autores em referência confirmaram essas previsões.

A Figura 1, ilustra a pressuposição básica do trabalho de Burgstahler e Dichev (1997). 


\section{Figura 1: Valor de Mercado de uma Ação como Função dos Lucros Esperados, Mantendo-se o Valor de Adaptação Constante}

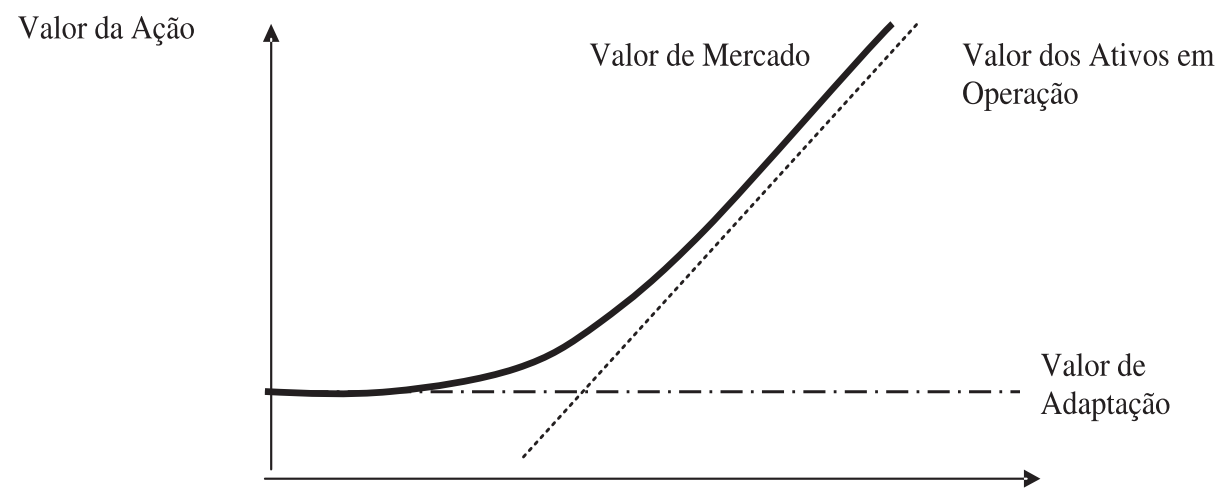

Lucros Esperados

A grande quantidade de abordagens utilizadas pelos trabalhos existentes sobre os determinantes do valor de mercado das empresas sugere que o tema se encontra aberto e, portanto, submeter proposições teóricas acerca desse tema a testes em ambientes distintos dos utilizados nos testes originais pode fornecer evidências empíricas que permitam, ou não, a sua generalização, agregando conhecimento sobre ele e auxiliando no direcionamento de novas pesquisas.

Cabe ressaltar que o Brasil se caracteriza como importante mercado emergente, que possui características institucionais e de mercado diferentes das do mercado americano, fonte dos dados utilizados nos testes empíricos realizados por Burgstahler e Dichev (1997), porquanto, dentre outras, podemos citar a título ilustrativo: tem seu sistema legal baseado no direito romano e não no direito consuetudinário, como é o americano; além disso predominam as empresas controladas por pequenos grupos fechados e pouco dependentes do mercado primário brasileiro de ações como fonte de financiamento (Lopes, 2002).

Outro aspecto a ser destacado refere-se ao fato de que os testes aqui realizados foram planejados e desenvolvidos de forma distinta da utilizada no trabalho de Burgstahler e Dichev (1997), podendo fornecer novas oportunidades de visualização das implicações relacionadas à teoria por eles testada. 


\section{A Pesquisa}

\section{Definição do Modelo e da Forma para a Realização dos Testes}

Com o objetivo de testar, com dados do mercado financeiro brasileiro, a principal predição do modelo de Burgstahler e Dichev (1997), que é sua convexidade, ou seja, (i) o valor da ação é função convexa do lucro, quando o valor patrimonial é mantido constante e, simultaneamente, (ii) o valor da ação é função convexa do valor patrimonial, quando o lucro é mantido constante; foi adotada como ponto de partida a equação (5) do trabalho supra-mencionado, que é apresentada a seguir:

$M V_{t}=\gamma_{1} B V_{t}+\gamma_{2} E_{t}+\varepsilon \quad$ equação (5) de Burgstahler e Dichev (1997)

onde: $\quad M V_{t}=P_{t} \Rightarrow$ preço da ação no instante $\mathrm{t}$ (market value)

$B V_{t}=V P A_{t} \Rightarrow$ valor patrimonial da ação no instante $\mathrm{t}$ (book value)

$E_{t}=L_{t} \Rightarrow>$ lucro por ação no instante t (earnings)

$\varepsilon=>$ termo de erro

$\gamma_{1}$ e $\gamma_{2}=>$ são coeficientes que se deseja obter

substituindo a nomenclatura para a lingua portuguesa, temos:

$P_{t}=\gamma_{1} V P A_{t}+\gamma_{2} L_{t}+\varepsilon$

Considerando-se que o banco de dados utilizado como fonte deste trabalho disponibiliza os valores de P/VPA (preço/valor patrimonial da ação), P/L (preço/ lucro da ação) e P (preço da ação), em dado dia, e que o uso de dados préformatados por um mesmo sistema aumenta a segurança quanto à consistência dos dados utilizados, e que P/VPA dividido por P/L gera a razão L/VPA (lucro/ valor patrimonial da ação), e ignorando-se o termo de erro, a equação acima pode ser escrita da seguinte forma:

$\frac{P_{t}}{V P A_{t}}=\gamma_{1} \frac{V P A_{t}}{V P A_{t}}+\gamma_{2} \frac{L_{t}}{V P A_{t}}$

que após os cancelamentos resulta em:

$\frac{P_{t}}{V P A_{t}}=\gamma_{1}+\gamma_{2} \frac{L_{t}}{V P A_{t}}$ 
onde: $\quad \gamma_{1}$ refere-se à interseção e define o coeficiente do VPA padronizado $\gamma_{2}$ refere-se ao coeficiente angular e define o coeficiente do L padronizado

A equação (1) acima equivale a uma padronização da equação (5) de Burgstahler e Dichev (1997) que, além de ser perfeitamente adaptada ao banco de dados disponível e permitir que a equação original de três dimensões seja testada em apenas duas, simplifica seu entendimento. No trabalho de Burgstahler e Dichev (1997) são realizadas as duas padronizações possíveis: a apresentada acima e a padronização em relação ao lucro; mas, como em ambos os casos o procedimento se resume em dividir todos os termos de uma equação por um mesmo número, os testes desenvolvidos neste trabalho consideram como ponto de partida apenas a equação (1) acima.

Os testes foram realizados através da comparação de duas carteiras: uma com baixa relação P/VPA e outra com alta relação P/VPA. A opção de se comparar duas carteiras e não dois grupos de ações se deve ao fato de que as carteiras, sendo representativas de suas ações componentes, reduzem a volatilidade apresentada e eliminam a necessidade de exclusão de outliers, outrossim permitem que sejam mantidos na pesquisa todos os tipos de empresas componentes da amostra e atenuam a não execução de ajustes individuais nos lucros dessas empresas no que tange aos itens extraordinários.

Para a comparação das duas carteiras foi utilizada a técnica de regressão linear com a inclusão de variáveis dummy para a diferenciação entre elas. Esta opção se deve ao fato de que os resultados oferecidos por esta técnica são mais completos e esclarecedores que os oferecidos pelos testes $\mathrm{F}$ normalmente utilizados em comparações.

Portanto os modelos utilizados no teste são dados por:

$$
\begin{aligned}
& \frac{P_{t}}{V P A_{t}}=\gamma_{1}+\gamma_{2} \frac{L_{t}}{V P A_{t}}+\gamma_{3} \text { dummy_Int }{ }_{\text {alto }} \\
& \frac{P_{t}}{V P A_{t}}=\gamma_{1}+\gamma_{2} \frac{L_{t}}{V P A_{t}}+\gamma_{3} \text { dummy_Int }_{\text {alto }}+\gamma_{4} \frac{L_{t}}{V P A_{t}} \text { dummy_Decl } \text { alto }_{\text {a }}
\end{aligned}
$$

onde os coeficientes das variáveis dummy mostram as diferenças no intercepto e na declividade entre as carteiras. 


\section{Formação das Carteiras, Dados sobre Ações e Período Abrangido pela Pesquisa}

Para definir o grupo de ações a ser considerado neste trabalho, algumas restrições foram aplicadas às ações negociadas na Bolsa de Valores de São Paulo com o objetivo de que a amostra utilizada fosse representativa do mercado como um todo. Carteiras representativas de mercado são utilizadas na composição de índices de mercado de ações, e para este trabalho foram utilizadas as carteiras do Índice Brasil - IBrX no período de janeiro de 1996 (primeira carteira existente para este índice) a janeiro de 2004, totalizando 8 anos ou 32 trimestres. O IBrX foi escolhido como base para este trabalho, por ser um dos três índices de ações mais importantes do mercado brasileiro, sendo mais amplo que o IBOVESPA e menos restritivo que o FGV-100. A carteira do IBrX é formada teoricamente por 100 ações, enquanto a carteira do IBOVESPA é formada por cerca de 50 ações e, por outro lado, o IBrX não restringe a participação de tipos específicos de empresas como ocorre no FGV-100, que exclui de sua carteira as ações de empresas estatais e de instituições financeiras.

A carteira teórica do IBrX é atualizada a cada quatro meses, sendo composta pelas 100 ações mais representativas entre as ações negociadas na Bovespa quanto a volume financeiro negociado e número de negócios. Essas ações são ponderadas na carteira do índice pelo seu respectivo número de ações disponíveis à negociação no mercado. Para fazer parte da carteira teórica do IBrX, é necessário também que a ação tenha sido negociada em pelo menos $70 \%$ dos pregões ocorridos nos doze meses anteriores à data da formação da carteira e que a empresa não esteja sob concordata preventiva, regime de falência ou com as negociações de suas ações suspensas por mais de 50 dias.

Devido ao grande número de fusões, aquisições e desmembramentos de empresas (por exemplo: Telebrás) ocorridos no período abrangido por este trabalho, e com o objetivo de garantir a estabilidade das carteiras utilizadas nos testes estatísticos aplicados, foi exigido que as ações componentes delas tenham feito parte da carteira de um determinado quadrimestre do início até o seu final.

As carteiras teóricas do $\mathrm{IBrX}$ foram obtidas diretamente na BOVESPA e os dados relativos às relações P/VPA e P/L trimestrais foram obtidos através do sistema de informações da Economática. Todos os dados de preço utilizados pelo sistema de informações da Economática para o cálculo dessas relações estavam devidamente ajustados quanto aos proventos distribuídos pelas empresas emissoras.

Para a obtenção das duas séries de carteiras utilizadas no teste, as ações componentes da amostra de cada trimestre foram classificadas em ordem 
decrescente, de acordo com a relação P/VPA, sendo as ações do quintil de mais alto P/VPA e as ações do quintil de mais baixo P/VPA representativas das respectivas carteiras de alto e baixo P/VPA. As ações que apresentaram valor patrimonial negativo foram excluídas das carteiras no período em que esse fato ocorreu. É importante observar que foram usados dados contemporâneos, e que nessa situação o valor do patrimônio líquido contém o lucro do período, porém esse fato é atenuado em razão dos dados serem trimestrais. Burgstahler e Dichev (1997, p. 195), trabalhando com dados anuais, relatam que nos testes que realizaram essa escolha operacional não afetou seus resultados.

\section{Desenvolvimento da Pesquisa e Resultados Empíricos}

Os dados iniciais da pesquisa, representados pelos pares ordenados (L/VPA, P/VPA) de 2667 (ação-trimestre) pontos relativos aos 32 trimestres abrangidos são apresentados na Figura 2, a seguir.

\section{Figura 2: Dados Originais}

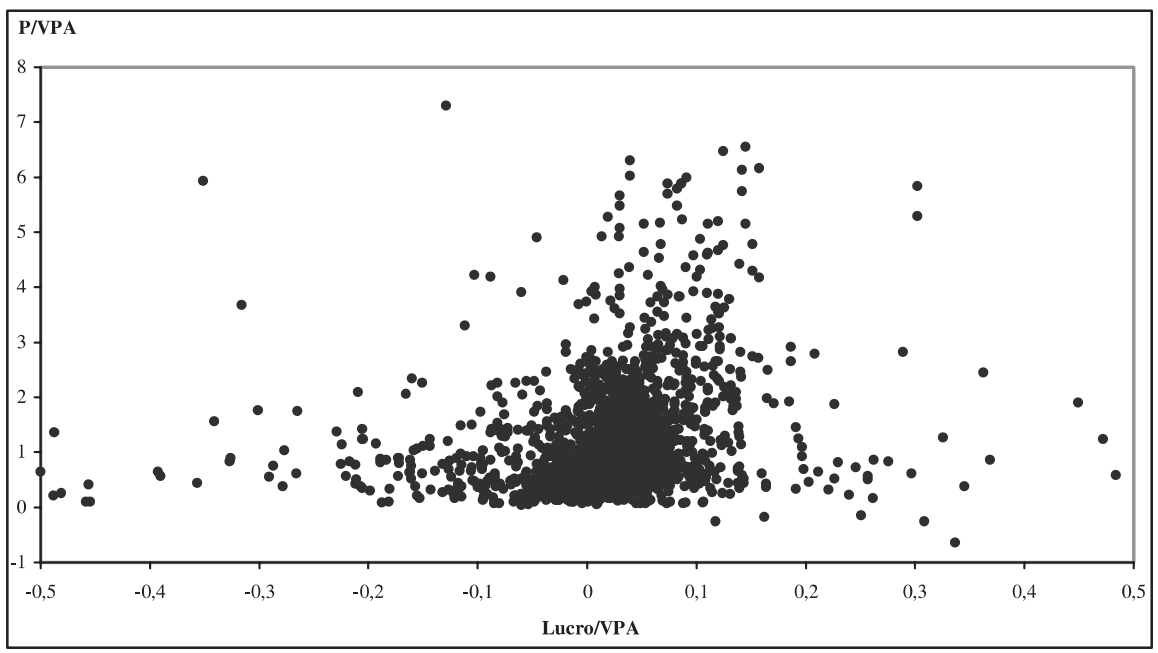

Na Figura 2, observa-se a existência de outliers e razões P/VPL negativas. Como esta pesquisa adota carteiras em lugar de ações individuais, o efeito dos outliers é atenuado. Já as ações com razões P/VPL negativas foram excluídas e não fazem parte das carteiras testadas. Observa-se também que a forma convexa prevista pelo modelo testado pode ser vislumbrada pela curvatura da densidade dos dados observada em torno do valor de L/VPA igual a zero. Considerando-se que no gráfico são plotados 2667 pontos, pode-se afirmar que razões L/VPA menores que 0,2 e maiores que 0,2 são claramente exceções, parecendo haver 
um limite em torno de 0,15 . Fica claro também que a grande maioria dos pontos se situa no intervalo entre -0,05 e 0,1. Quanto às razões P/VPA, observa-se que, acima de 3 , a frequiência cai acentuadamente.

A Figura 3, a seguir, apresenta a evolução ao longo do período abrangido pela pesquisa das relações L/P e P/VPA para as duas carteiras utilizadas.

\section{Figura 3: L/P e P/VPA das Carteiras}

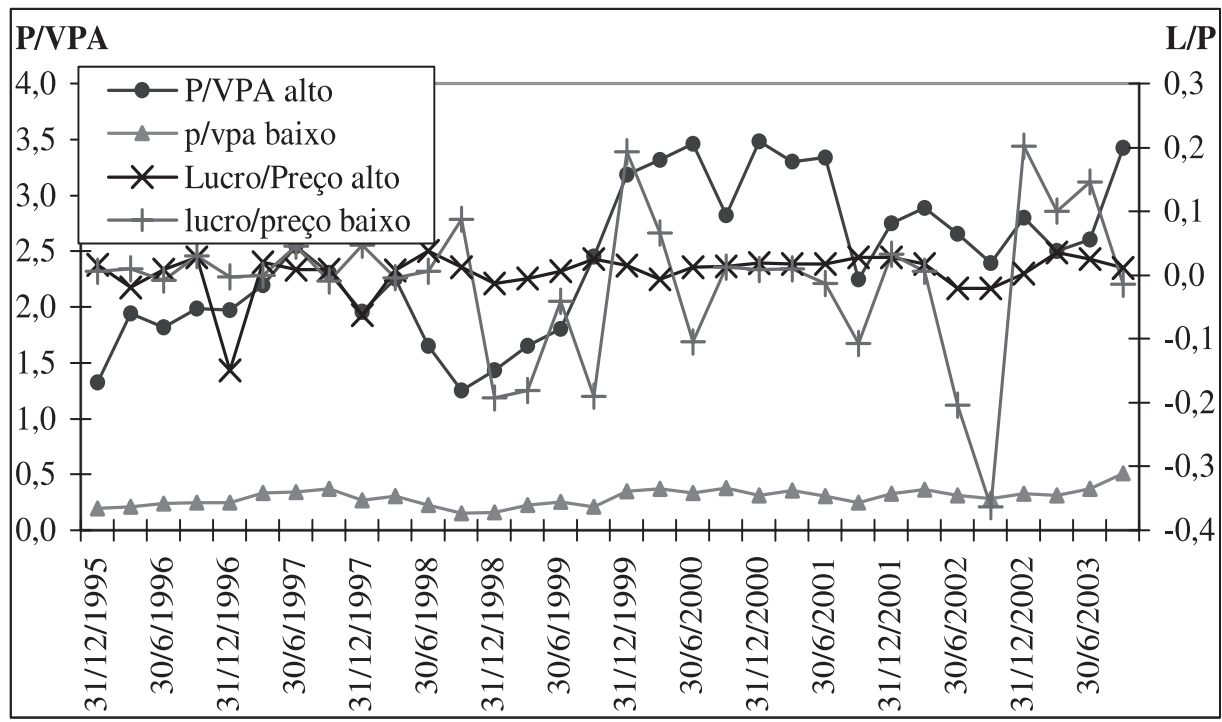

Na Figura 3 pode-se observar que, enquanto a carteira de baixa relação preço/ valor patrimonial (p/vpa baixo) permanece aproximadamente constante ao longo de todo o período abrangido pela pesquisa, a carteira de alta relação preço/ valor patrimonial (P/VPA alto) apresenta variações mais acentuadas. Quanto à relação lucro/preço, o comportamento das carteiras é o inverso, ou seja, enquanto a carteira de alta relação lucro/preço (L/P alto) permanece aproximadamente constante ao longo de todo o período abrangido pela pesquisa, a carteira de baixa relação lucro/preço (1/p baixo) apresenta variações mais acentuadas.

A Figura 4, apresenta o retorno acumulado das carteiras de alta e baixa relação P/VPA durante o período abrangido pela pesquisa. 
Figura 4: Retornos das Carteiras (base 100)

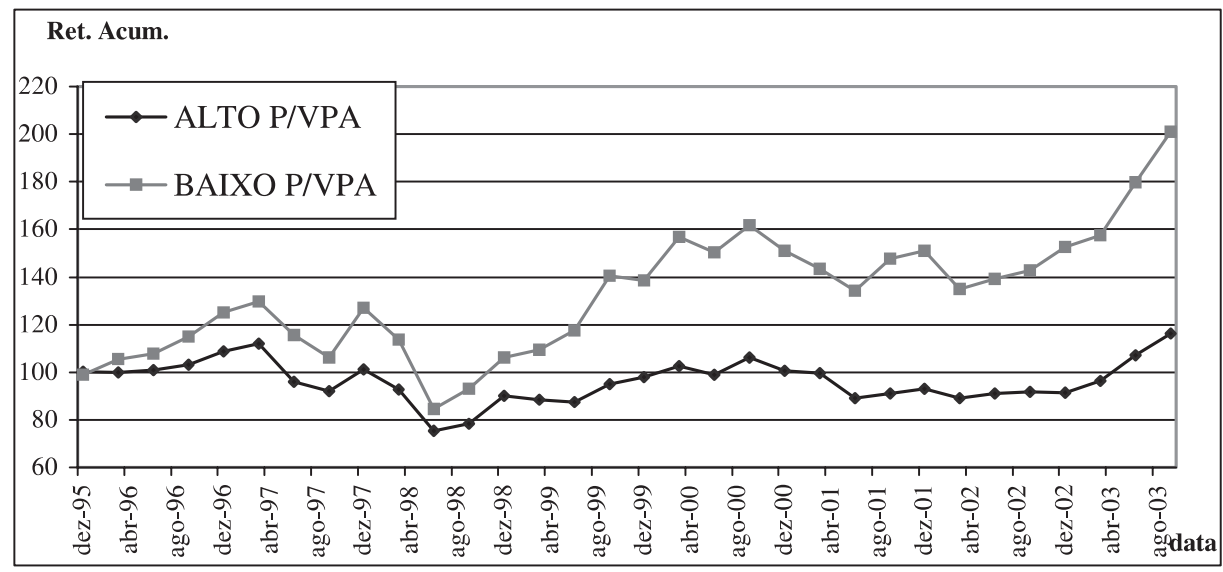

As Figuras 3 e 4 permitem a associação das razões contábeis P/VPA e L/P ao retorno das duas carteiras.

A Tabela 1, abaixo, apresenta as estatísticas descritivas relativas às duas carteiras que são o objeto dos testes aqui realizados. É importante notar que, quando se trabalha com carteiras, a obtenção de valores médios para a relação preço/lucro não tem sentido, por se tratar do inverso de uma proporção; por esta razão os valores reportados na Tabela 1 se referem aos da relação lucro/preço.

Tabela 1: Estatísticas Descritivas

\begin{tabular}{|c|c|c|c|c|}
\hline Carteiras & ALTO P/VPA & $\left(1^{\circ}\right.$ Quintil $)$ & BAIXO P/VPA & $\left(5^{\circ}\right.$ Quintil $)$ \\
\hline Estatística & P/VPA & L/P $\%$ & P/VPA & L/P \% \\
média & 2,4281 & $0,3763 \%$ & 0,2954 & $-1,3401 \%$ \\
desv.pad. & 0,6475 & $3,3792 \%$ & 0,0736 & $11,5398 \%$ \\
mediana & 2,4224 & $1,2519 \%$ & 0,3091 & $0,5865 \%$ \\
máximo & 3,4838 & $3,7313 \%$ & 0,5106 & $20,1395 \%$ \\
mínimo & 1,2488 & $-14,9466 \%$ & 0,1553 & $-36,3435 \%$ \\
\hline
\end{tabular}

A Figura 5, mostra a clara distinção entre as distribuições das 32 carteiras de alta e 32 carteiras de baixa relação P/VPA. Enquanto as carteiras de baixa relação P/VPA apresentam uma distribuição claramente horizontal, as carteiras de alta relação P/VPA apresentam uma distribuição com tendência vertical. 
Figura 5: Carteiras

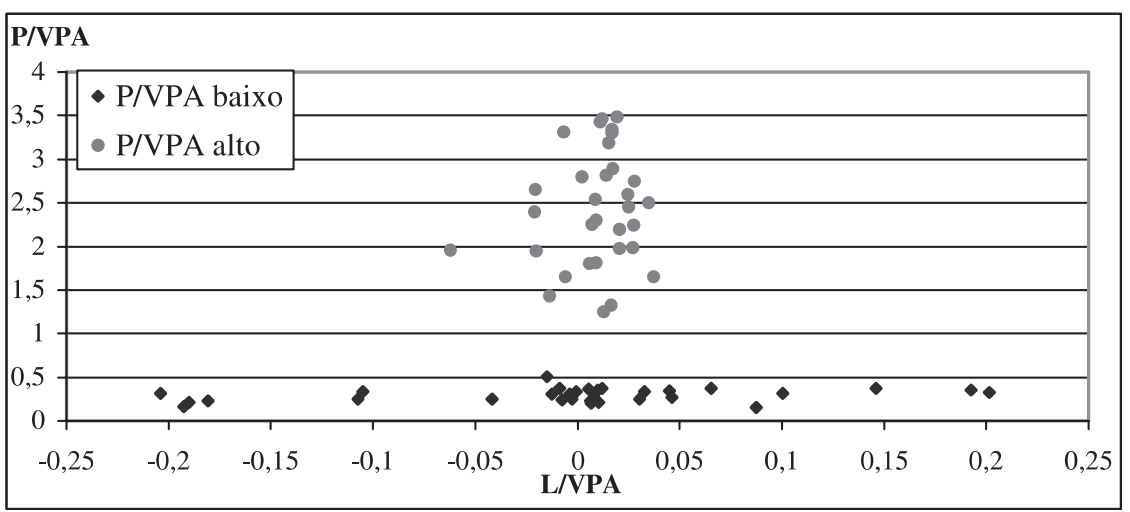

Os dados apresentados na Figura 5 claramente mostram que existe significativa diferença entre os interceptos e as declividades entre os dois grupos de dados.

Para a identificação da real diferença, e sua significância estatística, entre esses dois grupos de dados foram utilizadas a equações (2) e (3). A aplicação da técnica de regressão linear, segundo essas equações, mostrou a existência de autocorrelação serial nos resíduos. Por essa razão foi utilizada a técnica de Newey-West para correção de autocorrelação e heterocedasticidade (NeweyWest HAC Standard Errors \& Covariance). Esta técnica não altera os resultados dos coeficientes, mas ajusta os erros padrão e consequentemente as estatísticas t.

Como pode ser observado na Tabela 2, abaixo, a variável dummy relativa à declividade não apresentou significância, porém a variável dummy relativa ao intercepto é altamente significante, indicando uma real diferença entre as duas carteiras.

Tabela 2: Resultado das Regressões

\begin{tabular}{|c|c|c|c|c|c|c|}
\hline & Equação 2 & R2 ajustado & $=0,835$ & Equação 3 & R2 ajustado & $=0,836$ \\
\hline & coeficiente & estatística t & valor $\mathrm{p}$ & coeficiente & estatística t & valor $\mathrm{p}$ \\
\hline intercepto & 0,2968 & 17,71 & 0,0000 & 0,2964 & 16,44 & 0,0000 \\
\hline $\mathrm{L} / \mathrm{P}$ & 0,4289 & 2,330 & 0,0232 & 0,2462 & 2,78 & 0,0072 \\
\hline dummy_I_alto & 2,1273 & 10,773 & 0,0000 & 2,087 & 10,9 & 0,0000 \\
\hline dummy_D_alto & & & & 4,580 & 1,36 & 0,1779 \\
\hline
\end{tabular}


É interessante observar que, apesar da identificação de significativas diferenças entre os dois grupos de ações, o intercepto aumenta, quando se passa da carteira de baixa relação P/VPA para a de alta relação P/VPA. Observando o gráfico, é possível identificar que isso é decorrente da existência de um outlier $(\mathrm{L} / \mathrm{VPA}=$ 0,062) à esquerda do grupo que representa a carteira de alta relação P/VPA. Sem essa observação, os resultados seriam ainda mais próximos dos resultados previstos por Burgstahler e Dichev (1997), pois a variável dummy relativa à declividade provavelmente passaria a ser significante.

\section{CONCLUSÃo}

A grande quantidade de abordagens utilizadas pelos trabalhos existentes sobre os determinantes do valor de mercado das empresas sugere que o tema se encontra aberto e, portanto, submeter proposições teóricas sobre o tema a teste em ambientes distintos dos utilizados nos testes originais pode fornecer evidências empíricas que permitam, ou não, a sua generalização, agregando conhecimento sobre o tema e auxiliando no direcionamento de novas pesquisas.

A motivação inicial deste trabalho teve origem nos modelos de avaliação de ações que se baseiam em dados contábeis. O modelo de Ohlson (1995) prevê que o preço de mercado da ação de uma empresa é função linear aditiva do valor patrimonial e dos lucros anormais dessa empresa, enquanto o modelo de Burgstahler e Dichev (1997) prevê que o preço da ação de uma empresa é função convexa do valor patrimonial e dos lucros esperados para essa empresa. Este modelo (Burgstahler e Dichev) prediz, entre outras coisas que, quando a relação preço/valor patrimonial é baixa (alta), o valor da ação dependerá mais acentuadamente do valor patrimonial (lucro).

Este trabalho contribui para a literatura por trazer informações que podem auxiliar na compreensão das particularidades deste tema. Mais especificamente, o modelo teórico proposto por Burgstahler e Dichev (1997) se mostrou adequado aos dados utilizados nos testes empíricos aqui reportados. Esta contribuição somente foi possível pela aplicação do modelo de Burgstahler e Dichev (1997) à realidade brasileira, pois o mercado brasileiro é considerado emergente e possui características institucionais diferentes das do mercado americano, utilizado para os testes empíricos desenvolvidos pelos criadores do modelo que serviu de base para as formulações e testes desenvolvidos neste estudo.

Através do emprego de uma metodologia adaptada daquela utilizada no trabalho de Burgstahler e Dichev (1997), e da utilização de uma amostra composta por 
ações representativas do mercado brasileiro, os testes realizados por meio da comparação de duas carteiras de ações, uma de alta e outra de baixa razão preço/ valor patrimonial, geraram resultados que corroboram a relação predita pelos autores.

Como palavra final, é importante ressaltar que resultados empíricos, como os aqui reportados, devem ser interpretados com cuidado quanto a generalizações (Bamber et al., 2000).

\section{Artigo recebido em 19.02.2005. Aprovado em 03.08.2005.}

\section{AgradeCIMENTOS}

Alexsandro Broedel Lopes, que é pesquisador do CNPq, agradece o apoio recebido desse órgão.

\section{ReferênCias BibliográficAs}

Ball, R., \&

Brown, P. (1968).

An empirical evaluation of accounting income numbers. Journal of Accounting Research, 6(2), 159-178.

Bamber, L. S.,

Christensen, T. E., \&

Gaver, K. M. (2000).

Do we really 'know' what we think we know? A case study of seminal research and its subsequent overgeneralization. Accounting, Organizations and Society, 25(2), 103-129.

Barth, M. (1991).

Relative measurement errors among alternative pension asset and liability measures. The Accounting Review, 67(6), 433-463.

Barth, M.,

Beaver, W. H., \&

Landsman, W. (1992).

The market valuation implications of net periodic pension cost components.

Journal of Accounting and Economics, 15(2), 27-62.

Beaver, W. H. (1968).

The information content of annual earnings announcements. Journal of Accounting Research, 6(Supplement), 67-92.

Beaver, W. H. (1998).

Financial reporting: an accounting revolution (3rd ed.). Upper Saddle River, NJ: Prentice Hall.

Beaver, W. H.,

Clark, R., \&

Wright, W. (1979).

The association between unsystematic security returns and the magnitude of earnings forecast errors. Journal of Accounting Research, 17(2), 316-340. 
Bernard, V. (1995).

The Felthan-Ohlson framework: implications for empiricists. Contemporary Accounting Research, 11(2), 733-747.

Bernard, V. L., \&

Thomas, J. K. (1989).

Post-earnings-announcement drift: delayed price response or risk premium? Journal of Accounting Research, 27, 1-36.

Burgstahler, D. C. (1998).

Discussion of 'combining earnings and book value in equity valuation'. Contemporary Accounting Research, 15(3), 325-341.

Burgstahler, D. C., \&

Dichev, I. D. (1997).

Earnings, adaptation and equity value. The Accounting Review, 72(2), 187-215.

Collins, D., \&

Kothari, S. P. (1989).

An analysis of intertemporal and cross-sectional determinants of ERCs. Journal of Accounting and Economics, 11(2-3), 143-183.

Kormendi, R., \&

Lipe, R. (1987).

Earnings innovations, earnings persistence, and stock returns. Journal of Business, 60(3), 323-345.

Kothari, S. P. (2001).

Capital markets research in accounting. Journal of Accounting and Economics, 31(1-3), 105-231.
Kothari, S. P., \&

Zimmerman, J. L. (1995).

Price and return models. Journal of Accounting and Economics, 20(2), 155-192.

Landsman, W. (1986).

An empirical investigation of pension fund property rights. The Accounting Review, 61(4), 44-68.

Lee, C. M. C. (2001).

Market efficiency and accounting research: a discussion of 'capital market research in accounting' by $\mathrm{S}$. P. Kothari. Journal of Accounting and Economics, 31(1-3), 233-253.

Lipe, R. (1990).

The relation between stock returns and accounting earnings given alternative information. The Accounting Review, 65(1), 49-71.

Libby, R.,

Bloonsfield, R., \&

Nelson, M. W. (2002).

Experimental research in financial accounting. Accounting, Organisation and Society, 27(8), 775-810.

Lopes, A. B. (2001).

A relevância da informação contábil para o mercado de capitais: o modelo de Ohlson aplicado à BOVESPA. Tese de doutorado, Faculdade de Economia, Administração e Contabilidade, Universidade de São Paulo, SP, Brasil.

Lopes, A. B. (2002).

A informação contábil e o mercado de capitais. São Paulo: Pioneira Thomson Learning. 
Modigliani, F., \&

Miller, M.H. (1959).

The cost of capital, corporation finance and the theory of investments: reply. American Economic Review, 49(4), 655-669.

Modigliani, F., \&

Miller, M.H. (1961).

Dividend policy, growth, and the valuation of shares. The Journal of Business, 34(4), 411-433.

Nichols, D. C., \&

Wahlen, J. M. (2004).

How do earnings relate to stock returns? A review of classic accounting research with updated evidence. Accounting Horizons, 18(4), 263-286.

Ohlson, J. A. (1995).

Earnings, book values, and dividends in equity valuation. Contemporary Accounting Research, 11(2), 661-687.
Oliveira, E. F. (2001).

Contribuição à modelagem de avaliação de empresas em condições de risco. Tese de doutorado, Faculdade de Economia, Administração e Contabilidade, Universidade de São Paulo, SP, Brasil.

Penman, S. H. (1998).

Combining earnings and book value in equity valuation. Contemporary Accounting Research, 15(3), 291-324.

Watts, R. L., \&

Zimmerman, J. D. (1986).

Positive accounting theory. Upper Saddle River, NJ: Prentice Hall.

Wright, F. K. (1967).

Capacity of adaptation and the asset measurement problem. Abacus, 3(1), 74-79. 
